Enferm Bras 2020;19(4);317-28

https://doi.org/10.33233/eb.v19i4.4199

\title{
ARTIGO ORIGINAL \\ Assistência de enfermagem a queimados em hospital do nordeste brasileiro: estudo seccional
}

Izabelle Bezerra Costa*, Kezauyn Miranda Aiquoc*, Jessica Cristhyanne Peixoto Nascimento**, Rodrigo Rhuan Andrade Rocha ${ }^{\star \star \star}$, Rodrigo Assis Neves Dantas, D.Sc. ${ }^{* \star \star *}$, Daniele Vieira Dantas, D.Sc. ${ }^{* * \star}$

*Enfermeira, mestranda pelo Programa de de Pós-Graduação em Saúde Coletiva da UFRN, ${ }^{\star \star}$ Enfermeira, mestranda pelo Programa de Pós-Graduação em Enfermagem da UFRN,

***Graduando do Curso de Graduação em Enfermagem da UFRN, bolsista de iniciação científica PIBIC/UFRN, ${ }^{\star * * \star E n f e r m e i r o s, ~ p o ́ s-d o u t o r e s ~ p e l o ~ P r o g r a m a ~ d e ~ P o ́ s-G r a d u a c ̧ a ̃ o ~ e m ~ E n f e r m a g e m ~}$ da Universidade Federal de Sergipe (UFS) e professores adjuntos da Universidade Federal do Rio Grande do Norte (UFRN)

Recebido em 12 de junho de 2020; aceito em 24 de agosto de 2020.

Correspondência: Rodrigo Assis Neves Dantas, Rua Petra Kelly, 61, Condomínio Geraldo Galvão (casa 48), Nova Parnairim, 59152-330 Parnamirim RN

Izabelle Bezerra Costa: izabellebcosta@gmail.com

Kezauyn Miranda Aiquoc: kezauyn@gmail.com

Jessica Cristhyanne Peixoto Nascimento: jessicacristhy@gmail.com

Rodrigo Rhuan Andrade Rocha: rodrigo.andrade.rocha@hotmail.com

Rodrigo Assis Neves Dantas: rodrigoenf@yahoo.com.br

Daniele Vieira Dantas: daniele00@hotmail.com

\section{Resumo}

Objetivo: Caracterizar a assistência de enfermagem prestada às vítimas de queimaduras atendidas em um hospital de emergência no nordeste brasileiro. Métodos: Trata-se de um estudo epidemiológico com desenho seccional, individuado, observacional, transversal, de caráter descritivo e abordagem quantitativa conduzido mediante aplicação do checklist Strengthening the Reporting of Observational Studies in Epidemiology para número amostral correspondente a 144 indivíduos, no período de janeiro a junho de 2016. Resultados: No que concerne ao atendimento primário, identificou-se intubação, oxigenoterapia suplementar com técnica invasiva e não-invasiva, punção de acesso venoso periférico ou central e reposição eletrolítica com soro fisiológico $0,9 \%$ ou Ringer Lactato. Referente ao atendimento secundário, identificou-se intervenção cirúrgica e realização de curativos com Sulfadiazina de Prata 1\%, e/ou Kollagenase. Encontraram-se 36 diagnósticos de tipos distintos, porém no presente trabalho, em termos de representatividade, serão apresentados 22. Conclusão: A assistência pautou-se na intubação orotraqueal hospitalar; oxigenoterapia suplementar por cateter nasal; acesso venoso periférico para administração de soro fisiológico $0,9 \%$; manejo da dor com Diazepam; desbridamento cirúrgico e Sulfadiazina de Prata 1\% como cobertura das lesões. Dentre os diagnósticos de enfermagem, o risco de infecção foi o mais prevalente.

Palavras-chave: assistência hospitalar, epidemiologia, queimaduras.

\footnotetext{
Abstract

Nursing assistance to burns in a northeast Brazilian hospital: section study

Objective: To characterize the nursing care provided to burn victims treated at an emergency hospital in northeastern Brazil. Methods: This is an epidemiological study with a sectional, individual, observational, transversal design, of a descriptive character and a quantitative approach conducted by applying the checklist Strengthening the Reporting of Observational Studies in Epidemiology for a sample number corresponding to 144 individuals, from January to June 2016. Results: Regarding primary care, intubation, supplemental oxygen therapy with invasive and non-invasive technique, puncture was identified peripheral or central venous access and electrolyte replacement with $0.9 \%$ Saline or Ringer Lactate. Regarding secondary care,
} 
surgical intervention and dressing with $1 \%$ Silver Sulfadiazine and / or Kollagenase were identified. There were 36 diagnoses of different types, but in the present study, in terms of representativeness, 22 will be presented. Conclusion: Assistance was based on hospital orotracheal intubation; supplemental oxygen therapy by nasal catheter; peripheral venous access for administration of $0.9 \%$ Saline; pain management with Diazepam; surgical debridement and $1 \%$ Silver Sulfadiazine to cover the lesions. Among nursing diagnoses, the risk of infection was the most prevalent.

Keywords: hospital care, epidemiology, burns.

\section{Resumen \\ Asistencia de enfermería para quemaduras en un hospital brasileño del noreste: estudio de sección}

Objetivo: Caracterizar la atención de enfermería brindada a las víctimas de quemaduras tratadas en un hospital de emergencia en el noreste de Brasil. Métodos: Este es un estudio epidemiológico con un diseño transversal, individual, observacional, transversal, de carácter descriptivo y un enfoque cuantitativo realizado mediante la aplicación del checklist Strengthening the Reporting of Observational Studies in Epidemiology para un número de muestra correspondiente a 144 individuos, de enero a junio de 2016. Resultados: Con respecto a la atención primaria, intubación, oxigenoterapia suplementaria con técnica invasiva y no invasiva, se identificó la punción acceso venoso periférico o central y reemplazo de electrolitos con suero fisiológico al $0.9 \%$ o Ringer Lactato. Referente a la atención secundaria, se identificaron la intervención quirúrgica y el apósito con $1 \%$ de sulfadiazina de plata y / o Kollagenase. Hubo 36 tipos diferentes de diagnósticos, pero en el presente estudio, en términos de representatividad, se presentarán 22. Conclusión: La asistencia se basó en la intubación orotraqueal hospitalaria; oxigenoterapia suplementaria con catéter nasal; acceso venoso periférico para la administración de suero fisiológico al $0.9 \%$; manejo del dolor con Diazepam; desbridamiento quirúrgico y $1 \%$ de sulfadiazina de plata para cubrir las lesiones. Entre los diagnósticos de enfermería, el riesgo de infección fue el más frecuente.

Palabras-clave: atención hospitalaria, epidemiología, quemaduras.

\section{Introdução}

As lesões resultantes de queimaduras severas provocam intensa algia e sofrimento ao indivíduo, deixando sequelas de ordem psicológica, social, estética e funcional, muitas vezes irreversíveis, podendo levar o paciente ao óbito [1]. Estudo aponta que a gravidade desse trauma é definida de acordo com a avaliação da natureza do evento; do estado geral da vítima e das lesões - quanto ao agente causal, tempo de exposição, extensão, profundidade e localização [2].

Além disso, a qualidade do atendimento prestado impacta diretamente no agravamento do quadro do queimado e, consequentemente, no prognóstico da vítima [3]. Enquanto no contexto pré-hospitalar observa-se a indispensabilidade da avaliação primária referente a avaliação do estado geral da vítima conforme o mnemônico ABCDE do trauma (Airway, Breathing, Circulation, Disability e Exposure); na etapa secundária, as condutas são voltadas para o cálculo da Superfície Corporal Queimada (SCQ), realização de curativos e o transporte imediato para o hospital de referência [2].

Diante disso, o gerenciamento dos cuidados de enfermagem demanda preparo por parte do profissional que o desenvolve. Espera-se que o enfermeiro tenha habilidade para identificar as necessidades do paciente, elaborar o plano de cuidado, supervisionar sua execução e avaliar a efetividade de suas estratégias - a exemplo disso, destacam-se procedimentos que englobam desde a avaliação e execução de condutas iniciais no atendimento ao paciente até o gerenciamento da dor [4].

Não obstante, a análise dessas variáveis se faz necessária para elaboração e implementação de um tratamento eficaz para o cuidado ao paciente queimado - um desafio recorrente, tanto pela seriedade das lesões, quanto pelas inúmeras desordens sistêmicas como - choque séptico, complicações gastrintestinais, renais, cardiovasculares, adrenais, hematológicas e musculoesqueléticas que, usualmente, resultam em óbito [1]. Logo, o presente estudo se faz relevante no contexto da pesquisa epidemiológica em queimaduras, pois é necessária a compreensão sobre como a assistência de enfermagem é prestada à vítima de queimadura. 
Nessa perspectiva, o estudo objetiva caracterizar as vítimas de queimadura quanto ao perfil, a assistência prestada e os principais diagnósticos de enfermagem no processo de cuidar em um hospital de emergência no nordeste brasileiro.

\section{Material e métodos}

Trata-se de um estudo epidemiológico com desenho seccional, individuado, observacional, de dimensão temporal transversal, caráter descritivo e abordagem quantitativa, cujo cenário de realização se deu em um complexo hospitalar da região nordeste, referência no atendimento às vítimas de queimaduras cuja coleta de dados se deu durante o período de janeiro a junho de 2016.

No que concerne à condução da pesquisa, utilizou-se a diretriz Strengthening the Reporting of Observational studies in Epidemiology (STROBE) mediante o apontamento e checagem de vinte e dois itens considerados imprescindíveis para a execução de um estudo observacional de qualidade [5].

Para o desenvolvimento do estudo, realizou-se a construção do protocolo de pesquisa, e considerando os objetivos almejados, elaborou-se um instrumento para coleta de dados, que foi estruturado em quatro tópicos, a saber: caracterização sociodemográfica da vítima; aspectos clínicos da condição; caracterização do atendimento prestado e identificação dos diagnósticos de enfermagem presentes nos pacientes, utilizando a Taxonomia II da North American Nursing Diagnosis Association (NANDA) [6].

Sob essa perspectiva, o processo de amostragem se deu de forma não-probabilística por conveniência em meio a uma população de 225 internos, cuja amostra foi constituída por 144 pacientes - com erro amostral tolerável de $5 \%(0,05)$.

Para coleta de dados, foram eleitos dez acadêmicos de enfermagem da Universidade Federal do Rio Grande do Norte, componentes do Grupo de Pesquisa Núcleo de Estudos e Pesquisas em Urgência, Emergência e Terapia Intensiva (NEPET), os quais foram previamente treinados para realizar aplicação dos questionários e garantir a validade interna do estudo.

Após treinados, realizou-se a aplicação do estudo piloto com a finalidade de avaliar a adequação do instrumento para o alcance dos objetivos estabelecidos e ajustar aspectos deficientes para proceder a coleta definitiva.

No que se refere aos participantes do estudo, os critérios instituídos para inclusão na pesquisa foram: idade igual ou superior a 18 anos; estar consciente e consentir em participar da pesquisa ou ter sua participação autorizada pelo responsável mediante a assinatura do Termo de Consentimento Livre e Esclarecido (TCLE).

Os dados coletados foram digitados e organizados em planilha de dados eletrônica no Microsoft Excel 2016 e, posteriormente, inseridos no software Statistical Package for Social Science for Windows SPSS 20.0. Após a codificação e tabulação, os dados foram analisados por meio de estatística descritiva e os resultados apresentados em forma de tabelas e quadros.

Essa pesquisa cumpre todos os aspectos éticos presentes da Resolução n 466/2012, do Conselho Nacional de Saúde [7] e foi aprovada pelo Comitê de Ética em Pesquisa da Universidade Federal do Rio Grande do Norte, através do parecer no. 1.380.648.

Resultados

De acordo com a caracterização sociodemográfica das 144 vítimas de queimaduras, observou-se que 103 (71,5\%) eram do sexo masculino; $63(43,8 \%)$ apresentavam-se na faixa etária de 36 a 67 anos e 62 (43,1\%) abaixo de 35 anos; 62 (43,1\%) estavam solteiros; 24 (16,7\%) apresentavam $2^{\circ}$ grau completo, 23 (16\%) $1^{\circ}$ grau incompleto e $20(13,9 \%)$ não eram alfabetizados. Quanto à procedência, 91 (63,2\%) eram provenientes do interior do Estado e 35 $(24,3 \%)$ da capital.

A Tabela I apresenta a caracterização clínica quanto à natureza do evento. Observa-se que 69 (47,9\%) foram acidentes domésticos e 32 (22,2\%) acidentes no trabalho; sendo 55 $(38,2 \%)$ por chama direta e $30(20,8 \%)$ por escaldamento. Em relação a profundidade, $75(52,1 \%)$ tiveram queimaduras de $2^{\circ}$ grau; $46(31,9 \%)$ apresentaram extensão da superfície corporal queimada entre $10 \%$ e $20 \%$ e $42(29,2 \%)$ extensão maior que $30 \%$.

No que tange o atendimento secundário (Tabela II), 59 (41\%) realizaram intervenção cirúrgica, sendo 31 (21,5\%) desbridamento cirúrgico e 18 (12,5\%) enxertia; nos curativos, 99 $(68,8 \%)$ utilizaram Sulfadiazina de Prata 1\%, $25(17,4 \%)$ a combinação de Sulfadiazina de Prata $1 \%$ com Kollagenase e $16(11,1 \%)$ apenas Kollagenase. Constatou-se como principais regiões 
de desbridamento os membros superiores 31 (21,5\%), membros inferiores $29(20,1 \%)$ e tórax 19 $(13,2 \%)$; e no caso das enxertias foram os membros inferiores em oito $(5,6 \%)$ e superiores em seis $(4,2 \%)$.

Os sinais de infecção mais prevalentes diziam respeito a $32(22,2 \%)$ hipertermia, 11 $(7,6 \%)$ dor e $10(6,9 \%)$ leucocitose. A infecção em 23 (16\%), o óbito em quatro $(2,8 \%)$ e os problemas psiquiátricos em três $(2,1 \%)$ foram as principais complicações; as sequelas foram em $23(16 \%)$ vítimas de origens funcionais, $17(11,8 \%)$ estéticas e seis $(4,2 \%)$ os dois tipos.

Tabela I - Caracterização clínica das vítimas de queimaduras, quanto à natureza do evento, causa, profundidade e extensão corporal atingida da queimadura. Natal/RN, Brasil, 2016.

\begin{tabular}{|c|c|c|c|}
\hline Caracterização clínica & Variáveis & $\mathbf{n}$ & $\%$ \\
\hline \multirow[t]{6}{*}{ Natu reza do evento } & Adidentes domésticos & 69 & 47,9 \\
\hline & Acidentes de trabalho & 32 & 22,2 \\
\hline & Lesões autoinfligidas & 13 & 9,0 \\
\hline & Adidente de trânsito & 12 & 8,3 \\
\hline & Outros* & 12 & 8,3 \\
\hline & Não informado & 6 & 4,2 \\
\hline \multirow[t]{7}{*}{ Cau sa da queimadura } & Chama direta & 55 & 38,2 \\
\hline & Escaldamento & 30 & 20,8 \\
\hline & Agente químico & 24 & 16,7 \\
\hline & Eletricidade & 14 & 9,7 \\
\hline & Desconhecido & 10 & 6.9 \\
\hline & Superficie/Objeto aquecido & 9 & 6,3 \\
\hline & Superfície/Objeto resfriado & 2 & 1,4 \\
\hline \multirow[t]{8}{*}{ Profundidade } & $2^{\circ} \mathrm{grau}$ & 75 & 52,1 \\
\hline & $2^{\circ}$ e $3^{\circ}$ graus & 24 & 16,7 \\
\hline & $1^{\circ}$ e $2^{\circ}$ graus & 21 & 14,6 \\
\hline & $3^{\circ} \mathrm{grau}$ & 11 & 7,6 \\
\hline & Não identificado & 5 & 3,5 \\
\hline & $1^{\circ} \mathrm{grau}$ & 3 & 2,1 \\
\hline & $1^{\circ}, 2^{\circ}$ e $3^{\circ}$ graus & 3 & 2,1 \\
\hline & $4^{\circ} \mathrm{grau}$ & 2 & 1,4 \\
\hline \multirow{5}{*}{$\begin{array}{l}\text { Exten são corporal } \\
\text { atin gida }\end{array}$} & $10 \%$ a $20 \%$ & 46 & 31,9 \\
\hline & $<10 \%$ & 30 & 20,8 \\
\hline & $21 \%$ a $30 \%$ & 25 & 17,4 \\
\hline & $>30 \%$ & 42 & 29,2 \\
\hline & Não informado & 1 & 0,7 \\
\hline Total & & 144 & 100 \\
\hline
\end{tabular}

*Outros= agressão e fogos de artifícios; Fonte: elaborada pelos autores. 
Tabela II - Caracterização do atendimento primário quanto a intubação, oxigenoterapia, cateter venoso, reposição eletrolítica, monitorização hemodinâmica não-invasiva, avaliação neurológica, sondagens e sedação. Natal/RN, Brasil, 2016.

\begin{tabular}{|c|c|c|c|}
\hline Aten dimento primário & Variáveis & $\mathrm{n}$ & $\%$ \\
\hline \multirow[t]{2}{*}{ Intubação } & Realizado no Hospital & 4 & 2,8 \\
\hline & Realizado pelo SAMU & 2 & 1,4 \\
\hline \multirow[t]{5}{*}{ Oxigen oterapia suplementar } & Cateter nasal & 11 & 7,6 \\
\hline & Máscara de Venturi & 7 & 4,9 \\
\hline & Ventilação mecânica & 6 & 4,2 \\
\hline & BIPAP $^{*}$ & 2 & 1,4 \\
\hline & Bolsa-válvula-máscara & 1 & 0,7 \\
\hline \multirow[t]{2}{*}{ Cateter ven oso } & Periférico & 79 & 54,9 \\
\hline & Central & 10 & 6,9 \\
\hline \multirow[t]{4}{*}{ Reposição eletrolítica } & Soro fisiológico $0,9 \%$ & 59 & 41 \\
\hline & Ringer Lactato & 44 & 30,4 \\
\hline & Ringer Simples & 15 & 10,4 \\
\hline & Soro glicosado $5 \%$ & 5 & 3,5 \\
\hline \multirow{3}{*}{$\begin{array}{l}\text { Monitorização hemodinâmica não- } \\
\text { invasiva } \\
\text { Frequência cardíaca (bpm) }\end{array}$} & Normocardia & 58 & 40,3 \\
\hline & Taquicardia & 15 & 10,4 \\
\hline & Bradicardia & 3 & 2,1 \\
\hline \multirow[t]{3}{*}{ Pressão arterial $(\mathrm{mmHg})$} & Normotensão & 87 & 60,4 \\
\hline & Hipertensão & 21 & 14,6 \\
\hline & Hipotensão & 5 & 3,5 \\
\hline \multirow[t]{3}{*}{ Temperatura $\left({ }^{\circ} \mathrm{C}\right)$} & Normotermia & 66 & 45,8 \\
\hline & Hipertermia & 44 & 306 \\
\hline & Hipotermia & 2 & 1,4 \\
\hline \multirow[t]{3}{*}{ Frequếncia respiratória (mrpm) } & Eupneia & 52 & 36,1 \\
\hline & Taquipneia & 13 & 9 \\
\hline & Bradipneia & 1 & 0,7 \\
\hline Avaliação neu rológica & Normal (15) & 51 & 35,4 \\
\hline \multirow[t]{2}{*}{ ECG (3 a 15 pontos) } & Moderado (9 a 14) & 7 & 4,9 \\
\hline & Grave (3 a 8) & 4 & 2,8 \\
\hline \multicolumn{2}{|l|}{ Sonda vesical de demora } & 23 & 16 \\
\hline \multirow[t]{2}{*}{ Sonda nasogástrica/enteral } & Sonda nasoenteral & 11 & 7,6 \\
\hline & Sonda nasogástrica & 5 & 3,5 \\
\hline \multirow[t]{3}{*}{ Sedação } & Diazepam & 27 & 18,8 \\
\hline & Midazolan & 8 & 5,6 \\
\hline & Fentanil & 2 & 1,4 \\
\hline
\end{tabular}

*BI-level Positive Airway Pressure/Pressão positiva em vias aéreas a dois níveis; Fonte: elaborado por autores. 
Tabela III - Caracterização do atendimento secundário quanto à intervenção cirúrgica, curativos, desbridamento, enxertia, amputação, infecção, complicações e sequelas. Natal/RN, Brasil, 2016.

\begin{tabular}{|c|c|c|c|}
\hline Atendimento secundário & Variáveis & $\mathrm{n}$ & $\%$ \\
\hline \multirow[t]{6}{*}{ Intervenção cinúrgica } & Desbridamento cirúrgico & 31 & 21,5 \\
\hline & Enxertia & 18 & 12,5 \\
\hline & Curativo sob anestesia & 13 & 9 \\
\hline & Plástica & 12 & 8,3 \\
\hline & Correção de Fratura & 2 & 1,4 \\
\hline & Amputação & 2 & 1,4 \\
\hline \multirow{4}{*}{ Curativos } & Sulfadiazina de Prata $1 \%$ & 99 & 68,8 \\
\hline & Sulfadiazina de Prata $1 \%$ e Kollagenase & 25 & 17,4 \\
\hline & Kollagenase & 16 & 11,1 \\
\hline & Kollagenase e Cloranfenicol & 2 & 1,4 \\
\hline \multirow[t]{8}{*}{ Desbridamento } & MMSS & 31 & 21,5 \\
\hline & MMII & 29 & 20,1 \\
\hline & Tórax & 19 & 13,2 \\
\hline & Abdome & 14 & 9,7 \\
\hline & Face & 10 & 6,9 \\
\hline & Pescoco & 5 & 3,5 \\
\hline & Genitália & 3 & 2,1 \\
\hline & Cabeca & 2 & 1,4 \\
\hline \multirow[t]{6}{*}{ Enxertia } & MMII & 8 & 5,6 \\
\hline & MMSS & 6 & 4,2 \\
\hline & Face & 5 & 3,5 \\
\hline & Tórax & 5 & 3,5 \\
\hline & Pescoco & 2 & 1,4 \\
\hline & Abdome & 2 & 1,4 \\
\hline \multirow{9}{*}{ Sinal de infecção } & Hipertermia & 32 & 22,2 \\
\hline & Dor & 11 & 7,6 \\
\hline & Leucocitose & 10 & 6,9 \\
\hline & Sudorese & 6 & 4,2 \\
\hline & Exsudato purulento & 5 & 3,5 \\
\hline & Taquicardia & 4 & 2,8 \\
\hline & Edema & 3 & 2,1 \\
\hline & Fibrina & 2 & 1,4 \\
\hline & Hiperemia & 1 & 0,7 \\
\hline \multirow[t]{6}{*}{ Complicaçỏes } & Infeccão & 23 & 16 \\
\hline & Óbito & 4 & 2.8 \\
\hline & Problemas psiquiátricos & 3 & 2,1 \\
\hline & Congestão pulmonar & 2 & 1,4 \\
\hline & Int oxicação muscarínica & 1 & 0,7 \\
\hline & Necrose & 1 & 0,7 \\
\hline \multirow[t]{3}{*}{ Sequelas } & Funcionais & 23 & 16 \\
\hline & Estéticas & 17 & 11,8 \\
\hline & Estéticas e funcionais & 6 & 4,2 \\
\hline
\end{tabular}

Fonte: elaborado por autores.

Após a análise dos diagnósticos de enfermagem, encontraram-se 36 tipos distintos, porém, no presente trabalho, em termos de representatividade, serão apresentados 22 (Tabela IV). Dentre esses, quinze são reais e sete de risco, conforme a Taxonomia II da NANDA [6]. Esses dados revelaram a prevalência do risco de infecção 99 (68,8\%), a integridade da pele prejudicada $89(61,8 \%)$ e a dor aguda $85(59 \%)$. Vale salientar que em uma mesma vítima pôde ser identificado mais de um diagnóstico. 
Tabela IV - Diagnósticos de enfermagem presentes nos pacientes vítimas de queimaduras atendidas no complexo hospitalar referência para atendimento de queimados. Natal/RN, Brasil, 2016.

\begin{tabular}{lll}
\hline Diagnóstico de enfermagem & $\mathrm{n}$ & $\%$ \\
\hline Risco de infecção & 99 & 68,8 \\
Integridade da pele prejudicada & 89 & 61,8 \\
Dor aguda & 85 & 59 \\
Risco de choque & 29 & 20,1 \\
Mobilidade física prejudicada & 21 & 14,6 \\
Risco de desequilíbrio do volume de líquidos & 17 & 11,8 \\
Hipertermia & 17 & 11,8 \\
Risco de disfunção neurovascular periférica & 14 & 9,7 \\
Baixa autoeșima situacional & 13 & 9 \\
Distúrbio da imagem corporal & 11 & 7,6 \\
Padrão de sono prejudicado & 10 & 6,9 \\
Padrão respiratório ineficaz & 8 & 5,6 \\
Risco de violência direcionada a si mesmo & 8 & 5,6 \\
Automutilação & 8 & 5,6 \\
Risco de aspiração & 7 & 4,9 \\
Perfusão tissular periférica ineficaz & 7 & 4,9 \\
Função motora prejudicada & 6 & 4,2 \\
Conforto prejudicado & 5 & 3,5 \\
Déficit no autocuidado para banho & 4 & 2,8 \\
Ansiedade & 3 & 2,1 \\
Volume de líquidos deficiente & 3 & 2,1 \\
Risco de queda & 3 & 2,1 \\
\hline
\end{tabular}

Fonte: elaborado por autores.

Discussão

Os principais achados do presente estudo demonstram uma predominância de vítimas do sexo masculino. A masculinidade, historicamente, está associada ao comportamento agressivo ou imprudente [8] e é percebida como inerente à natureza masculina e necessária para manutenção da imagem de poder [9]. Logo, esse comportamento pode explicar o maior risco de acidentes, sobretudo com queimaduras.

Além disso, a maioria das vítimas de queimaduras estavam na faixa etária abaixo de 35 anos e entre 36 e 67 anos, atingindo assim parte de uma população economicamente ativa, gerando problemas de ordem econômica e social [10]. Característica também identificada em estudo realizado no Malawi, o qual destacou também o predomínio masculino e o quanto gerou de prejuízo financeiro familiar, sobretudo devido à necessidade de afastamento da atividade laboral $[10,11]$. Ademais, ao retornar a rotina de trabalho, pode surgir sentimentos de culpa, pois as sequelas das queimaduras prejudicam o seu desempenho, o que pode gerar profunda tristeza [12]. Nesse aspecto, as consequências da queimadura atingem não só o corpo, mas também a saúde mental das vítimas.

No que tange ao local da ocorrência, estudos demonstram que os acidentes domésticos e de trabalho apresentam elevada incidência $[13,14]$. Ao analisar esse dado associado ao nível de escolaridade, evidencia-se a necessidade do ensino de práticas de prevenção e primeiros socorros em queimaduras, sobretudo, à população com baixa escolaridade [1,10], além de treinamento contínuo sobre segurança do trabalho a fim de mitigar esses acidentes [15]. Já no que diz respeito aos agentes etiológicos, as queimaduras por chama direta e por escaldamento foram predominantes, corroborando os demais estudos [16,17].

Quanto a profundidade das lesões, a maioria das vítimas apresentaram queimaduras de $2^{\circ} \mathrm{grau}$. Em estudo realizado em Fortaleza, destacou-se que $80 \%$ das vítimas atendidas durante a pesquisa apresentavam essa mesma característica [18]. Os autores afirmam que queimaduras nessas dimensões atingem estruturas importantes que podem acarretar sequelas funcionais importantes, além de estéticas [18].

No que concerne ao atendimento à vítima de queimadura, a priorização das condutas segue o mesmo critério de qualquer vítimas de trauma, cujo foco está em manter vias aéreas pérvias e controle da cervical, permitir ventilação, garantir a circulação, avaliar as condições neurológicas e controlar o ambiente - tais condutas objetivam estabilizar o paciente, reduzir as complicações provenientes do trauma e diminuir seu tempo de internação [2]. 
Sobre o procedimento de intubação orotraqueal, observou-se um pequeno número de pacientes submetidos a ele em ambiente pré-hospitalar. Contudo, autores reforçam a importância de se reduzir a intubação desnecessária, pois procedimentos invasivos aumentam o risco de infecção [17]. Outrossim, em ambiente extra-hospitalar, a implementação das vias aéreas artificiais no paciente traumatizado, quando não haja a viabilidade de respiração por outros métodos, apresentam vantagens como a diminuição significativa do risco de aspiração, possibilidade de aspiração profunda da traqueia, prevenção de insuflação gástrica e viabilidade de administração de medicamento através do dispositivo [19].

O emprego de oxigenoterapia suplementar ocorreu principalmente através do cateter nasal. Sob esse aspecto, estudo que analisou as intervenções de emergência realizadas nas vítimas de trauma evidenciou que a oxigenoterapia administrada em vítimas de queimadura ou politraumatizadas se deve ao grande potencial de descompensação hemodinâmica [19]. Assim, recomenda-se a administração de oxigênio umidificado por esse dispositivo restringindo a intubação a casos de insuficiência respiratória aguda e situações que possam evoluir para edema tardio com obstrução das vias respiratórias, tornando complicado a intubação [20].

A solução de soro fisiológico a $0,9 \%$ foi a mais empregada na reposição eletrolítica inicial, divergindo dos achados da literatura que recomendam a solução de Ringer Lactato isotônico, e o hipertônico em casos de choque hipovolêmico e SCQ superior a 40\% [2,3]. Outro estudo aponta a fluidoterapia aquecida como método empregado para viabilizar a expansão intravascular transitória e estabilizar o volume vascular associado a perda de fluidos no paciente traumatizado. Contudo, sua prescrição não deve ser realizada de forma indiscriminada tendo em vista que pode agravar hemorragias devido ao aumento da pressão arterial e hemodiluição [19].

A monitorização hemodinâmica não-invasiva foi realizada em 125 (86,8\%) das vítimas; e os parâmetros prevalentes encontrados: a taquicardia, hipertensão, hipertermia e taquipneia. Uma falha encontrada no atendimento prestado está relacionada a qualidade dessa monitorização, constatando lacunas na aferição de alguns sinais, similar a estudo de mesma metodologia [21].

Destaca-se que essa conduta deve ser executada com critérios rígidos, tendo em vista que a monitorização é uma ferramenta fundamental no reconhecimento de situações potencialmente perigosas, em tempo hábil para se estabelecer uma terapêutica adequada e imediata [3,21]. Logo, deve-se estimular essa avaliação hemodinâmica contínua para definição e acompanhamento da evolução do quadro clínico da vítima de queimadura [21].

Realizou-se também a aplicação da Escala de Coma de Glasgow (ECG), predominando a classificação de 15 pontos, indicando o estado de consciência e responsividade pela maioria das vítimas [21]. De acordo com estudo realizado na Alemanha, com vítimas de trauma, evidenciou-se sobre a importância da aplicação adequada dessa escala e do teste de reatividade pupilar uma vez que são ferramentas importantes nos prognósticos e parâmetros de monitoramento da vítima de trauma [22]. Os autores demonstraram, ainda, que o somatório da pontuação da ECG acima de 8 pontos está relacionada a baixa probabilidade de mortalidade [22], o que pode explicar também a baixa taxa de mortalidade, nos achados do presente estudo, uma vez que o número de vítimas com os resultados da pontuação da ECG abaixo de 8 pontos coincidiram com o número de óbito.

Em relação à farmacologia empregada, a droga mais administrada foi o Diazepam, seguido do Midazolam, que apresenta início de ação mais rápido. No que concerne ao controle da dor e sedação do paciente crítico, a farmacologia empregada objetiva promover a redução do consumo de oxigênio pelo miocárdio, além de reduzir do tempo de internamento hospitalar. Contudo, é necessário que as doses sejam ajustadas corretamente a fim de evitar a ocorrência de agravos associados a doses inadequadas desses fármacos e seus efeitos ao organismo paciente [23].

Além disso, a infecção consistiu na principal complicação apresentada pelas vítimas e a hipertermia foi o principal sinal indicativo no caso desses pacientes. Isso se relaciona com a SCQ e a profundidade das queimaduras, podendo evoluir com septicemia, assim como as repercussões sistêmicas [24,25]. Em estudo de caso, foi evidenciado que não só a lesão poderia aumentar o risco de infecção, mas também os inúmeros procedimentos que essa cliente sofre durante o processo de hospitalização e nas trocas dos curativos [25]. Com isso, para proteção dele é importante que a equipe de enfermagem sistematize seu cuidado, difunde conhecimento sobre segurança do paciente e elabore instrumentos que operacionalizam o cuidado a fim de minimizar esses riscos [25].

As sequelas mais prevalentes foram as funcionais. Nesse aspecto, a depender do local, a correção torna-se mais complicada devido à anatomia presente, o que infere na função 
desempenhada pela determinada região [1]. Isso pode levar ao afastamento temporário ou permanente de determinadas atividades, impactando diretamente na qualidade de vida da vítima [1].

Sob a perspectiva dos Diagnósticos de Enfermagem, o mais prevalente foi o de Risco de Infecção sendo acompanhado, em segundo plano, pelo de Integralidade da Pele Prejudicada. Tais julgamentos se baseiam na ruptura da principal barreira entre agentes patogênicos e corrente sanguínea: a pele. Destaca-se, ainda, como fator propiciador à infecções a permanência do paciente em um ambiente hospitalar permeado por agentes infecciosos exógenos [26].

Outro ponto pertinente, refere-se à proporcionalidade direta entre tamanho da superfície corporal queimada e taxas de morbimortalidade do indivíduo acometido [26]. Em corroboração a esse achado, foi observado que fatores inerentes à clínica dos pacientes queimados como complexidade das queimaduras, comprometimento respiratório, instabilidade hemodinâmica, isquemia tecidual e aumento da resposta inflamatória favorecem a ocorrência de Infecções Relacionadas a Assistência por Staphylococcus aureus resistente à meticilina [27].

Já no que tange ao diagnóstico de dor aguda, estudo realizado na China aponta que com a mudança no trabalho de enfermagem, sendo cada vez mais voltado às necessidades do paciente, requer desse profissional uma atenção mais assertiva e proativa quanto a esse acometimento $[28,29]$. Por ser uma vítima que além de apresentar dor aguda no início, posteriormente dor intensa e um longo período com esse agravo e suas complicações, grandes cargas de estresse e ansiedade podem ser geradas, inclusive na equipe [28]. Logo, o manejo adequado está diretamente relacionado ao sucesso do tratamento e fortalecimento do vínculo com a equipe, sendo imprescindível no processo de cicatrização e qualidade de vida [30].

Nesse aspecto, vale salientar o uso da terapia não-farmacológica como um importante adjuvante à ação farmacológica para gerenciamento da algia na vítima de queimadura, visto que - controle inadequado desta interfere significativamente na condição clínica do indivíduo acometido. Diante disso, a literatura aponta inúmeras vantagens dos métodos não farmacológicos para o tratamento da dor aguda no paciente queimado, dentre elas destacam-se: baixo custo, fácil implementação, boa aceitação por parte dos clientes e resultados significativos na quebra do ciclo dor-ansiedade [31].

Na queimadura, infelizmente, há uma centralidade no que concerne aos prejuízos físicos. No entanto, como aponta a literatura, os traumas psicológicos podem acarretar danos por toda a vida $[32,33]$. Sob essa perspectiva, o presente estudo apresentou como os diagnósticos de enfermagem, dos quais revelam prejuízos à saúde mental, foram: baixa autoestima, distúrbio da imagem corporal, padrão de sono prejudicado, ansiedade, risco de violência direcionada a si e automutilação.

Nesse sentido, o estudo evidencia a imprescindibilidade de direcionar os cuidados não só para o que tange a integridade da pele prejudicada, mas também para a saúde mental desses sobreviventes. Em um ensaio clínico randomizado, realizado no Irã, apostaram no empoderamento psicossocial das vítimas. Por meio da educação multimídia, considerada também como a mais eficaz na educação em saúde de vítimas com baixa escolaridade, a intervenção foi realizada com o objetivo de capacitar a vítima para lidar com sua nova realidade, além de possibilitar a observar e resolver seus problemas psicológicos. Com isso, foi observado que as vítimas, comparadas àquelas que tiveram somente a educação em saúde- sem 0 empoderamento psicossocial- tiveram melhoras na sua qualidade de vida, autoestima e autonomia [33].

Com relação às limitações do estudo, observou-se a necessidade de padronização na coleta de dados, quanto aos termos e parâmetros utilizados no preenchimento do instrumento de coleta. Além disso, foram constatadas lacunas na monitorização hemodinâmica não-invasiva, relacionadas a aferição ausente ou incompleta.

Diante desses resultados supracitados, conclui-se que o atendimento primário aos pacientes queimados caracterizou-se por: intubação orotraqueal mais prevalente no ambiente hospitalar; oxigenoterapia suplementar através de cateter nasal; acesso venoso periférico como via de administração prioritária para a solução de soro fisiológico 0,9 \% e Ringer Lactato, respectivamente.

Quanto a monitorização hemodinâmica não-invasiva, os parâmetros anormais prevalentes foram taquicardia, hipertensão, hipertermia e taquipneia; a ECG retratou estado de 
consciência e responsividade pela maioria das vítimas. No manejo da dor e ansiedade os fármacos mais empregados foi Diazepam, seguido do Midazolam.

No atendimento secundário, ressaltam-se as intervenções de desbridamento cirúrgico e a enxertia, realizados em membros superiores e inferiores; nos curativos a Sulfadiazina de Prata $1 \%$ foi mais utilizada. A infecção preponderou dentre as complicações, apresentando a hipertermia como principal sinal indicativo. As sequelas de caráter funcional prevaleceram. Dentre os diagnósticos de enfermagem, o Risco de infecção, a Integridade da pele prejudicada e a Dor aguda, predominaram.

Por fim, é importante ressaltar que a enfermagem se posiciona mais próxima do paciente, logo, esse tipo de estudo gera uma fonte de dados para a constante atualização técnica e científica desses profissionais, provendo ao paciente uma terapêutica individualizada e integrada. Além disso, essa caracterização da assistência prestada reforça a necessidade da implementação de protocolos de atendimento, tratamento e cuidados aos pacientes queimados que certamente contribuirão para assegurar a qualidade da assistência para esta população.

1. Li H, Yao Z, Tan J, Zhou J, Li Y, Wu J et al. Epidemiology and outcome analysis of 6325 burn patients: a five-year retrospective study in a major burn center in Southwest China Sci Rep 2017;7:46066. https://doi.org/10.1038/srep46066

2. National Association of Emergency Medical Technicians. American College of Surgeons. Atendimento Pré-hospilatar ao Traumatizado. $8^{\underline{a}}$ ed. Rio de Janeiro: Elsevier; 2016.

3. Vivó C, Galeiras R, Caz MD. Initial evaluation and management of the critical burn patient. Med Intensiva 2016;40(1):49-59. https://doi.org/10.1016/i.medine.2016.01.002

4. Secundo CO, Silva CCM, Feliszyn RS. Protocolo de cuidados de enfermagem ao paciente queimado na emergência: Revisão integrativa da literatura. Rev Bras Queim 2019;18(1):39-46. http://www.rbqueimaduras.com.br/details/458/pt-BR

5. Malta M, Cardoso LO, Bastos FI, Magnanini MMF, Silva CMFP. Iniciativa STROBE: subsídios para a comunicação de estudos observacionais. Rev Saude Pública 2010;44(3):559-65. https://doi.org/10.1590/S0034-89102010000300021

6. Diagnóstico de enfermagem da NANDA: definições e classificação 2015-2017. Porto Alegre: Artmed; 2015.

7. Brasil. Ministério da Saúde. Conselho Nacional de Saúde. Resolução n 466 de 12 de dezembro de 2012. Normas para pesquisa envolvendo seres humanos. Brasília: Ministério da Saúde; 2012.

8. Drumond EF, Souza HNF, Hang-Costa TA. Homicídios, álcool e drogas em Belo Horizonte, Minas Gerais, Brasil, 2000-2009. Epidemiol Serv Saúde 2015;24(4):607-16. https://doi.org/10.5123/S1679-49742015000400003

9. Dagirmanjian FB, James RM, Justin B, Alexander C, Joseph D, Anthony P et al. How do men construct and explain men's violence? J Interpers Violence 2017;32(15):227597. https://doi.org/10.1177/0886260515625511

10. Santos GP, Freitas NA, Bastos VD, Carvalho FF. Perfil epidemiológico do adulto internado em um centro de referência em tratamento de queimaduras. Rev Bras Queim 2017;16(2):81-86. http://www.rbqueimaduras.com.br/details/367/pt-BR/perfilepidemiologico-do-adulto-internado-em-um-centro-de-referencia-em-tratamento-dequeimaduras

11. Barnett, BS, Mulenga M, Kiser MM, Charles AG. Qualitative analysis of a psychological supportive counseling group for burn survivors and families in Malawi. Burns 2017;43;602-7. https://doi.org/10.1016/j.burns.2016.09.027

12. Kornhaber R, Childs $C$, Cleary M. Experiences of guilt, shame and blame in those affected by burns: a qualitative systematic review. Burns 2018;44(5);1026-39. https://doi.org/10.1016/j.burns.2017.11.012

13. Echevarría-Guanilo ME, Gonçalves N, Farina JA, Rossi LA. Avaliação da qualidade de vida relacionada à saúde no primeiro ano após a queimadura. Esc Anna Nery Rev Enferm 2016;20(1):155-66. https://doi.org/10.5935/1414-8145.20160021

14. Fan X, Ma B, Zeng D, Fang X, Li H, Xiao S, Wang G et al. Burns in a major burns center in East China from 2005 to 2014: Incidence and outcome. Burns 2017;43(7):1586-95. https://doi.org/10.1016/j.burns.2017.01.033 
15. Dissanaike S, Ha D, Mitchell D, Larumbe E. Socioeconomic status, gender, and burn injury: A retrospective review. Am J Surg 2017;214(4):677-81. https://doi.org/10.1016/j.amjsurg.2017.06.012

16. Barcellos LG, Silva APP, Piva JP, Rech L, Brondani TG. Características e evolução de pacientes queimados admitidos em unidade de terapia intensiva pediátrica. Rev Bras Ter Intensiva 2018;30(3):333-7. https://doi.org/10.5935/0103-507x.20180045

17. Arruda FCF, Castro BCO, Medeiros JF, Valadão WJ, Reis GMD. Análise epidemiológica de 2 anos na Unidade de Queimados do Hospital de Urgências Governador Otávio Lage de Siqueira, Goiânia, Brasil. Rev Bras Cir Plást 2018;33(3):389-94. https://doi.org/10.5935/2177-1235.2018RBCP0152

18. Santos GP, Freitas NA, Bastos VD, Carvalho FF. Perfil epidemiológico do adulto internado em um centro de referência em tratamento de queimaduras. Rev Bras Queimaduras 2017;16(2):81-86. http://www.rbqueimaduras.com.br/details/367/ptBR/perfil-epidemiologico-do-adulto-internado-em-um-centro-de-referencia-emtratamento-de-queimaduras

19. Schweitzer G, Nascimento ERP, Nascimento KC, Moreira AR, Amante LN, Malfussi LBH. Intervenções de emergência realizadas nas vítimas de trauma de um serviço aeromédico. Rev. bras. enferm.2017;70(1):54-60. https://doi.org/10.1590/0034-71672016-0311

20. Cai AR, Hodgman El, Kumar PB et al. Evaluating pre burn center intubation practices: an update. J Burn Care Res 2017;38(1):e23-e29. https://doi.org/10.1097/BCR.0000000000000457

21. Praça WR, Brandão MC, Magro MCS, Hermann PRS. Perfil epidemiológico e clínico de vítimas de trauma em um hospital do Distrito Federal. Rev. Prev. Infecç. Saúde. 2017; 3(1):1-7. http://www.ojs.ufpi.br/index.php/nupcis/article/ view/6219/pdf

22. Emami $P$, Czorlich $P$, Fritzsche FS, Westphal M, Rueger JM, Lefering $R$ et al. Impact of Glasgow Coma Scale score and pupil parameters on mortality rate and outcome in pediatric and adult severe traumatic brain injury: a retrospective, multicenter cohort study. J Neurosurg 126(3):760-7. https://doi.org/10.3171/2016.1.JNS152385

23. Nascimento BKS, Lima LMM, Silva NF, Henriques FVCA, Almeida TSO, Freire RHL et al. A utilização de drogas sedativas em pacientes críticos submetidos à ventilação mecânica: uma revisão integrativa dos achados clínicos. Braz J Health Review 2019;2(4):2948-60. https://doi.org/10.34119/bjhrv2n4-059

24. Hernández CMC, Núñez VP, Doural KG, Machado AAB. Características de crianças hospitalizadas por queimaduras em um hospital em Manzanillo, Cuba. Rev Bras Queimaduras 2017;16(3):169-73. http://www.rbqueimaduras.com.br/details/389/ptBR/caracteristicas-de-criancas-hospitalizadas-por-queimaduras-em-um-hospital-emmanzanillo-cuba.

25. Belba MK, Petrela EY, Belba AG. Epidemiology and outcome analysis of sepsis and organ dysfunction/failure after burns. Burns 2017;43(6):1335-47. https://doi.org/10.1016/j.burns.2017.02.017

26. Palmieri TL. Infection prevention: unique aspects of burn units. Surg Infect 2019;20(2):111-4. https://doi.org/10.1089/sur.2018.301

27. Kim JJ, Blevins MW, Brooks DJ, Stehle JR Jr, McLouth CJ, Viviano JP, Holmes Iv JH, Bischoff WE. Successful control of a methicillin-resistant Staphylococcus aureus outbreak in a burn intensive care unit by addition of universal decolonization with intranasal mupirocin to basic infection prevention measures. Am J Infect Control 2019;47(6):661-5. https://doi.org/10.1016/j.ajic.2018.11.016

28. Jiang XS, Xu L, Li L, Zhang LQ. Investigation and analysis of the difference between the nursing needs of adult burn patients and nurses' cognition. Zhonghua Shao Shang Za Zhi 2018;20;34(10):731-5. https://doi.org/10.3760/cma.j.issn.1009-2587.2018.10.016

29. Paz F, Rego LP. Dificuldades da assistência de enfermagem diante do quinto sinal vital: a dor. Enferm Bras 2012;11(5):309-13. https://doi.org/10.33233/eb.v11i5.3822

30. Paggiaro AO, Silva-Filho MLe, Carvalho VF, Castro GLG. Manejo da dor em crianças queimadas: Revisão integrativa. Rev Bras Queimaduras 2018;17(2):123-31. http://www.rbqueimaduras.com.br/details/439/pt-BR/manejo-da-dor-em-criancasqueimadas--revisao-integrativa

31. Costa IB, Dantas DV, Dantas RAN, Oliveira SP, Aiquoc KM, Sarmento SDG. Terapias não farmacológicas para o manejo da dor em vítima de queimaduras: uma revisão sistemática. Revista Inspirar: movimento e saúde 2019;19(2):1-20. 
https://www.inspirar.com.br/revista/terapias-nao-farmacologicas-para-o-manejo-da-dorem-vitima-de-queimaduras-uma-revisao-sistematical

32. Zamanzadeh V, Valizadeh L, Lotfi M, Salehi F. Preserving self-concept in the burn survivors: a qualitative study. Indian J Palliat Care 2015;21(2):182-91. https://doi.org/10.4103/0973-1075.156492

33. Mamashli L, Ardebili FM, Ghezeljeh TN, Manafi F, Mehri B. Investigating the psychosocial empowerment interventions through multimedia education in burn patients. World J Plast Surg 2019;8(3):372-81. https://doi.org/10.29252/wjps.8.3.372 